\title{
Phase transitions in Ising model induced by weight redistribution on weighted regular networks
}

\author{
Menghui Li, ${ }^{1,2}$ Ying Fan, ${ }^{1}$ Jinshan $\mathrm{Wu},{ }^{3}$ and Zengru Di ${ }^{* 1}$ \\ ${ }^{1}$ Department of Systems Science, School of Management, \\ Center for Complexity Research, Beijing Normal University, Beijing 100875, P.R.China. \\ ${ }^{2}$ Temasek Laboratories, National University of Singapore, 117508, Singapore \\ ${ }^{3}$ Department of Physics \& Astronomy, University of British Columbia, Vancouver, B.C. Canada, V6T $1 Z 1$.
}

\begin{abstract}
In order to investigate the role of the weight in weighted networks, the collective behavior of the Ising system on weighted regular networks is studied by numerical simulation. In our model, the coupling strength between spins is inversely proportional to the corresponding weighted shortest distance. Disordering link weights can effectively affect the process of phase transition even though the underlying binary topological structure remains unchanged. Specifically, based on regular networks with homogeneous weights initially, randomly disordering link weights will change the critical temperature of phase transition. The results suggest that the redistribution of link weights may provide an additional approach to optimize the dynamical behaviors of the system.
\end{abstract}

Pacs: 89.75.Hc, 05.70.Fh, 64.60.Fr

Keywords: Weighted Network; Ising Model; Phase Transition;

\section{INTRODUCTION}

Complex networks are widely used to describe the interaction structure of many complex systems. Since the Watts-Strogatz (WS) model[1] was proposed, the structure, function, and evolution of complex networks are investigated extensively. In particular, many kinds of dynamical processes evolving on networks are studied, e.g., the spread of infectious disease 1], fast response and coherence of Hodgkin-Huxley neurons 2], percolation 3], security of system (Cascade-based attacks) [4], phase transitions [5, [6], focusing on the influence of topological structure of the underlying network on the dynamical behaviors. All these studies try to understand the role of topology, usually by disordering the links. For instance, based on regular networks, one can construct small-world networks by rewiring or adding links randomly. For disorder of the rewiring type, the nearest neighbor links are rewired with the rewiring probability $p_{r}$ to form random shortcut links 1]. For disorder of the adding type, without removing local links, random shortcut links are added with the probability $p_{a}[7,8]$ or with the probability $P(l) \sim l^{-\delta}$, where $l$ is the Euclidean distance between two vertices [9]. Both types display phase transitions from regular networks to small-world networks by varying the single parameter $p_{r}$ or $p_{a}$ representing disorder.

The behaviors of dynamics on small-world networks are usually compared to those on regular networks to understand the role taken by network topologies. In this paper, we focus on the phase transition of the Ising models on weighted networks $[6]$.

The phase transition of the Ising model on smallworld networks was investigated extensively in the past

\footnotetext{
*Author for correspondence: zdi@bnu.edu.cn
}

years. Barrat and Weigt [5] and Gitterman [10 studied the crossover from one-dimensional to mean-field behavior for the ferromagnetic Ising model, which presents a phase transition of mean-field type for any value of the rewiring probability $p_{r}>0$. Later, Herrero 11] investigated the ferromagnetic transition of the Ising model on small-world networks generated by rewiring two-dimensional and three-dimensional lattices. In the thermodynamic limit, the phase transition has a meanfield character for any finite value of the rewiring probability $p_{r}$. In Ref. 12], D. Jeong et al studied the Ising model on small-world networks with the coupling strength $J_{i j}$ decaying algebraically with the distance $r$, i.e., $J_{i j}(r) \propto r_{i j}^{-\alpha}$, where $r_{i j}$ is the geometrical distance between two vertices $i$ and $j$ on the underlying onedimensional lattice. Das and Sen [13] studied the quenching dynamics of the ferromagnetic Ising model on densely connected small-world networks at zero temperature. Recently, Chatterjee and Sen [14] investigated the critical behavior of the Ising model on one-dimensional networks, where long-range bonds were taken into consideration with the probability $P(l) \sim l^{-\delta}$ if distances $l>1$. In addition, the self-averaging properties of the Ising model on networks were also investigated recently, such as the magnetization $M$ and the susceptibility $\chi[15]$.

Most of the above studies of Ising models on networks have mainly concentrated on disordering the topological structure of regular lattices. In fact, link weights, directly related to the coupling strength of spins, can also affect the phase transition of the Ising model. For instance, the topology-dependent coupling strength, e.g. $J_{i j} \propto\left(k_{i} k_{j}\right)^{-\mu}$, had remarkable influence on the phase transition of the Ising model[16]. In the case that the coupling strength is directly related to the distance $d_{i j}$ between vertices, e.g. $J_{i j} \propto d_{i j}^{-\alpha}$, where $d_{i j}$ depends on link weights on the path, an interesting question is: How does randomizing link weights affect the process of phase 
transition of the Ising model?

In many realistic networks besides the connecting structure (described by links), interaction intensity (described by link weights) is also an important property of networks, and usually plays an important role as to the dynamical processes evolving on the networks. For example, the number of passengers or flights between any two airports in airport networks [17, 18], the closeness of any two scientists in scientific collaboration networks 18 21], and the reaction rates in metabolic networks 22 are all crucial to characterize the corresponding systems. Recently more and more studies in complex networks focus on the effect of link weights on dynamics, such as epidemic spread [23], transportation [24 26], percolation [27], synchronization 28 30], functional organization 31] and so on.

For weighted networks, disordering link weights provides another way to adjust the structure and to optimize the dynamical behaviors of networks. In our previous papers, we introduced one method to disturb the weightlink correspondence 32 and another mechanism to redistribute link weights [33], and investigated their influences on properties of networks. With randomly redistributed link weights, the average path length decreases, while the average clustering coefficient increases [33]. This indicates that random redistribution of link weights may induce small-world phenomena. In addition, redistributing link weights can enhance synchronizability of chaotic maps on weighted regular networks [33, 34]. In this paper, we consider Ising spins on the vertices of weighted regular networks and investigate the effects of weights redistribution on the phase transition.

This paper is organized as follows. In Section II we will briefly introduce the method of disordering link weights. Then, in Section III, we will give the results of the Ising model on weighted regular networks. We find that disordering link weights has significant influence on the critical temperature of phase transition, which reflects the important role taken by the link weights in networks. Finally, in Section [V] some concluding remarks are given.

\section{THE METHOD OF DISORDERING LINK WEIGHTS}

In weighted networks, link weights can be represented as measures of dissimilarity or similarity. For dissimilarity weight $w$, e.g., the distance between two airports, the distance between two vertices, which are connected by a third vertex and two links (with dissimilarity weights $w_{1}$ and $w_{2}$ respectively), is defined as $d=w_{1}+w_{2}$. For similarity weight $\tilde{w}$, e.g. the number of cooperations between any two scientists in scientific collaboration networks or the coupling strength between oscillators, the similarity distance between two vertices, which are connected by a third vertex and two links (with similarity weights $\tilde{w}_{1}$ and $\tilde{w}_{2}$ respectively), is defined as $\tilde{w}=1 /\left(\frac{1}{\tilde{w_{1}}}+\frac{1}{\tilde{w_{2}}}\right)$, which is smaller than both $\tilde{w}_{1}$ and $\tilde{w}_{2}$. Distance can be defined as the inverse of similarity weight, i.e. $d=1 / \tilde{w}$. In the following discussion, the dissimilarity weight is chosen as $w_{i j} \in[1, \infty)$, and consequently the similarity weight $\tilde{w}_{i j}=1 / w_{i j}$ is in $(0,1][33]$.

Our initial setup is a ring lattice with $N$ vertices. Every vertex links to $k$ nearest neighbors. Each link has the same dissimilarity weight, e.g. $w=10$, which corresponds to the distance. All connections are undirected. We assume that there is a minimum unit of weight, e.g., $\Delta w=1$. The procedures of disordering link weights are as follows:

1. Every unit of weight in the original lattice is removed with probability $P$ from the original link, and transferred to a link randomly chosen over the whole lattice.

2. Step 1 is repeated until each unit of weight in the original lattice has been tried once. The reallocated weights will not be considered again.

3. If the unit of weight being selected is the last unit left on that link, it will not be moved. This is to avoid disconnecting the link so that the topology remains unchanged.

Without changing the binary structure, above procedures allow us to adjust the network with uniform link weight $(P=0)$ to the one with poisson weight distribution $(P=$ $1)$. We are able to discuss and compare quantities such as distance and clustering coefficients for networks before and after disordering link weights. In our calculation, $\tilde{w} \in(0,1]$, we revise the definition of weighted clustering coefficient [35, 36] as follows(see details in Ref. [33]),

$$
C_{H}^{w}(i)=\frac{\sum_{j, k} \tilde{w}_{i j} \tilde{w}_{j k} \tilde{w}_{k i}}{\sum_{j, k} \tilde{w}_{i j} \tilde{w}_{k i}}
$$

and

$$
C_{O}^{w}(i)=\frac{2}{k_{i}\left(k_{i}-1\right)} \sum_{j, k}\left(\tilde{w}_{i j} \tilde{w}_{j k} \tilde{w}_{k i}\right)^{1 / 3} .
$$

With disordering link weights, the average path length $L(P) / L(0)$ decreases, while the average clustering coefficient $C(P) / C(0)$ increases(as shown in Fig 1). This demonstrates that besides rewiring links, disordering link weights also leads to small-world phenomena. This provides a potential approach to optimize the dynamical behaviors of system.

In the following section, we will investigate the Ising models on weighted regular networks with the coupling strength $J_{i j}$ decaying algebraically with distance $d_{i j}$, where $d_{i j}$ represents the shortest distance between two vertices $i$ and $j$. By investigating system behaviors in the intermediate region $0<P<1$, we can study the effect of disordering link weights on the phase transition of Ising models. 


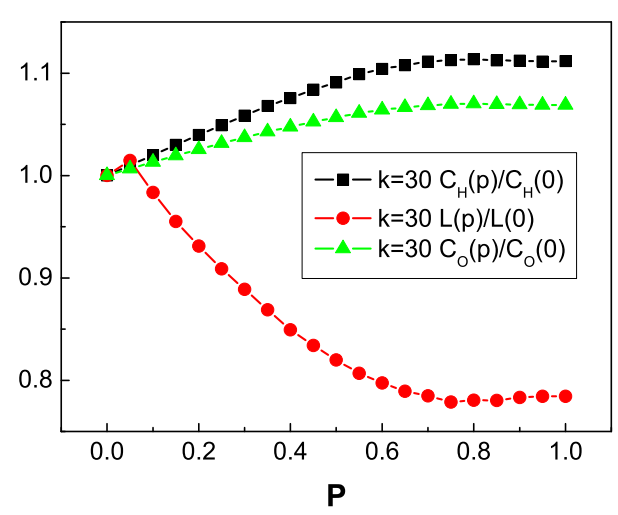

FIG. 1: Without rewiring links, characteristic path length $L(P) / L(0)$ and clustering coefficient $C(P) / C(0)$ for the family of randomly weight redistributed networks $(N=300, k=$ 60, $w=10$ ). $C_{H}$ and $C_{O}$ are defined in Eq.(1) and Eq.(2) respectively. The $x$-axis is the probability of redistributing link weights, and the $y$-axis is the value of $L(P) / L(0)$ and $C(P) / C(0)$, where $L(0)$ and $C(0)$ are the values of initial uniform regular networks. All results are averaged over 20 random realizations of the disordering process and the relative standard deviation is less than $2 \%$.

\section{ISING MODEL ON WEIGHTED REGULAR NETWORKS}

We perform Monte Carlo (MC) simulations with the heat bath algorithm at various values of $P$. The Hamiltonian for an Ising model on weighted regular networks is given by

$$
H=-\frac{1}{2} \sum_{i \neq j} J_{i j} \sigma_{i} \sigma_{j},
$$

where $\sigma_{i}(= \pm 1)$ is the Ising spin on vertex $i$. The distance-dependent interaction $J_{i j}$ reads

$$
J_{i j}=d_{i j}^{-\alpha},
$$

where $d_{i j}$ is the shortest path length (rather than the geometrical distance) between two vertices $i$ and $j$. The role of $\alpha$ has been investigated in Ref. 37, 38], indicating that (i) mean-field-type critical behavior for $1<\alpha<1.5$; (ii) continuously varying critical exponents for $1.5<\alpha<$ 2; (iii) for $\alpha=2$ a hybrid transition, with a jump in magnetization but continuous energy is expected, and the short-range interaction regime with no phase transition for $\alpha>2$. In this paper, we mainly focus on the influence of redistributing link weights on the phase transition of the Ising model, so we only investigate the mean field type, e.g. $\alpha=1$ for simplification. Although the value $\alpha=1$ is somewhat singular, as there are stability issues (e.g. in the thermodynamic limit the ground state energy per site is infinite), in our simulations these singularities do not show up, and we assume that the model behaves like a regular model with mean-field exponents, which corresponds to our numerical findings.

For each realization of networks, every vertex is given a spin $\left(\sigma_{i}=1\right.$ or $\left.\sigma_{i}=-1\right)$ randomly with equal probability, so the magnetization is nearly zero at the beginning (paramagnetic phase). Then, the system evolves according to the metropolis algorithm.

The following quantities are computed on weighted regular networks after the system reaches equilibrium:

1. Magnetization per spin

$$
M=\sum \sigma_{i} / N
$$

2. Binders fourth-order cumulant

$$
U=1-\frac{\left\langle m^{4}\right\rangle}{3\left\langle m^{2}\right\rangle^{2}}
$$

3. Susceptibility per spin is calculated from the fluctuation of the order parameter:

$$
\chi=\frac{N}{K_{B} T}\left(\left\langle m^{2}\right\rangle-\langle m\rangle^{2}\right),
$$

where $K_{B}$ is the Boltzmanns constant.

4. Specific heat capacity per spin is obtained from the energy fluctuations at a given temperature

$$
C=\frac{\left\langle H^{2}\right\rangle-\langle H\rangle^{2}}{N\left(K_{B} T\right)^{2}}
$$

Here $\langle\cdots\rangle$ denotes the thermal average, taken over 3000 Monte Carlo steps after discarding 3000 Monte Carlo steps for equilibration at each temperature. All results are averaged over 20 different network realizations and over 10 random realizations of the redistribution process, respectively.

The simulations are made on a ring of typically $N=$ 300 vertices with $k=60$ neighbors per vertex, and last until the system reaches a stationary state, where the magnetization fluctuates around the average value. When $P=0$, link weights are all homogeneous, while for $P \neq 0$ link weights are heterogeneous. After disordering link weights, the average shortest path length decreases clearly(Fig,1). Figure 2 presents the quantities as the functions of temperature on weighted regular networks with $P=0, P=0.2$ and $P=1.0$. For different $P$, the curves of magnetization are significantly different. With the increasing of $P$, the critical temperature moves to higher values (as shown in Fig 2 (a)). Correspondingly, other curves are also quite distinct for different $P$, such as Binder's fourth-order cumulant, Susceptibility per spin and Specific heat capacity per spin. The appearance of the susceptibility peak in Fig:2 (c) as well as the specific heat peak in Fig 2 (d) unanimously suggests that the phase transition emerges at a finite temperature. At 

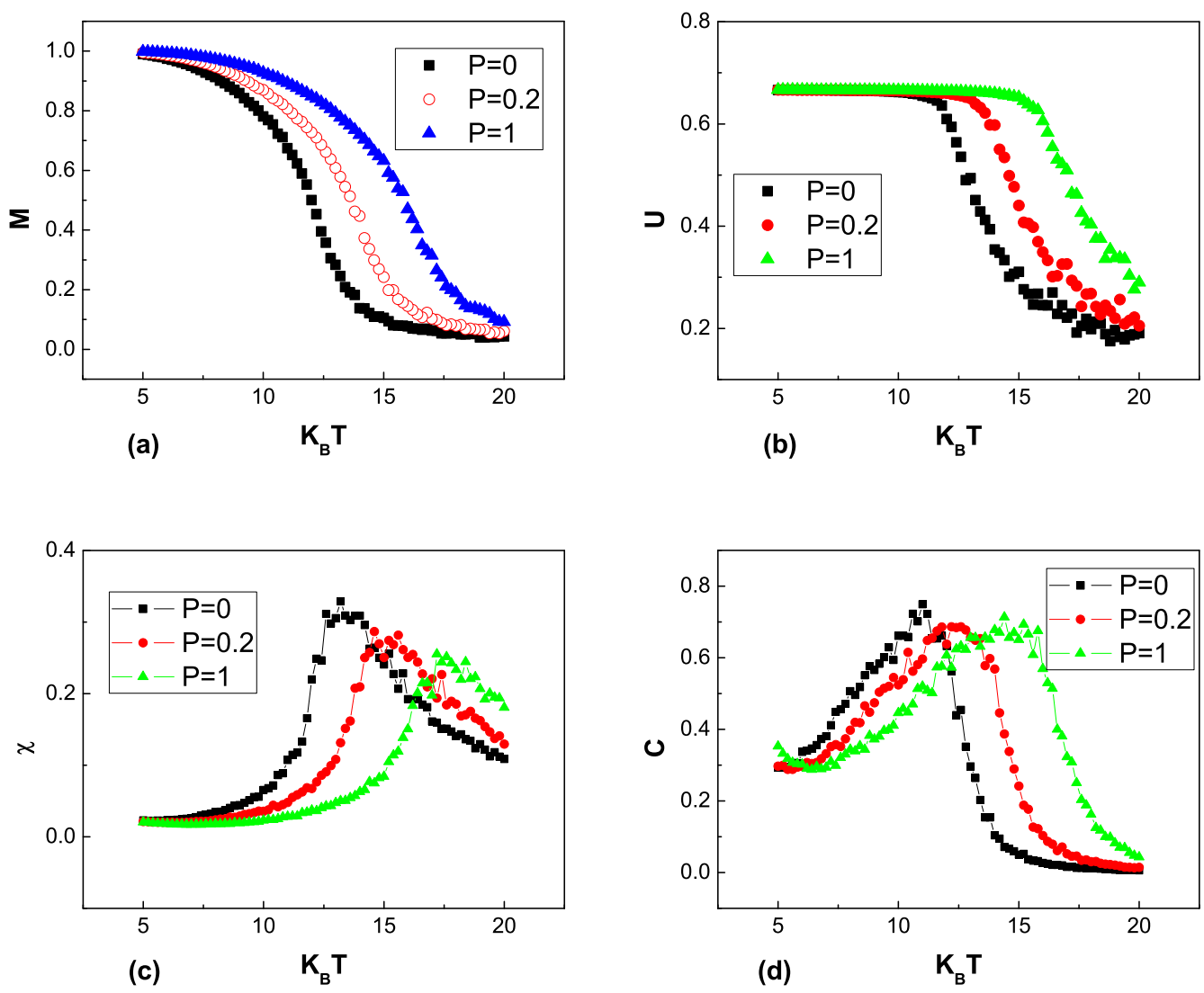

FIG. 2: Quantities of Ising model versus temperature $K_{B} T$ on weighted regular networks with interaction $J_{i j}=d_{i j}^{-1}$ for $P=0,0.2,1$. (a) Magnetization per spin $M$, (b) Binders cumulant $U$, (c) Susceptibility per spin $\chi$, (d) Specific heat capacity per site $C$.

temperatures where $\chi$ and $C$ display peaks, the fluctuations are very large. These give some information that critical phenomena could be observed at the peak temperatures. It can be seen clearly (Fig 2 (c)) that when the disordering probability $(P)$ increases, the peak temperature of the susceptibility $\chi$ keeps increasing while the peak value of $\chi$ keeps decreasing.

In numerical simulations, it is very difficult to determine the critical temperature $T_{c}$. Instead of $T_{c}$, we will observe the peak temperature $T_{p}$ of the susceptibility. Figure 3 gives the dependence of the peak temperature on the disordering probability $P$, indicating that the peak temperature increases with the disordering probability $P$ raising. The peak temperature as a function of the disordering probability $P$ is given by a power law

$$
T_{p} \sim P^{\beta},
$$

where $\beta \simeq 0.10$. This indicates that disordering link weights has diminishing marginal effects. With the raising of disordering probability $P$, it has smaller effect on the shift of the peak temperature.

At a fixed temperature, the value of order parameter $M$ increases as the disordering probability $P$ raises (as

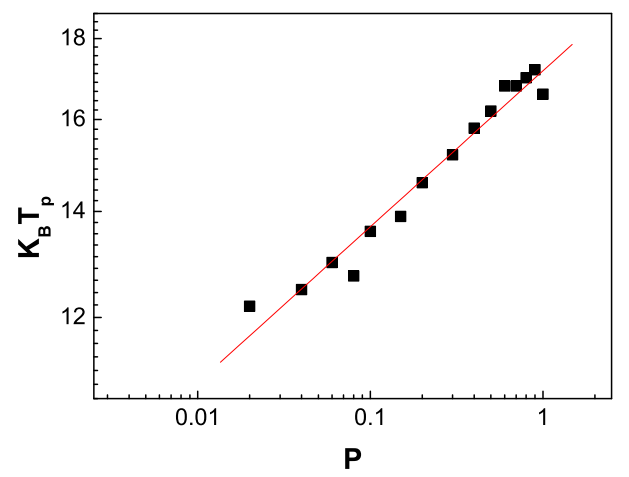

FIG. 3: Peak temperature $T_{P}$ versus the probability $P$ of disordering link weights on the log-log plane. The line is the power fit.

shown in Fig. (4). Fortunately, the magnetization grows rapidly around $T_{p}$. This implies that there is no doubt about the onset of the ordering.

Since the system is too small, the phase transition at 


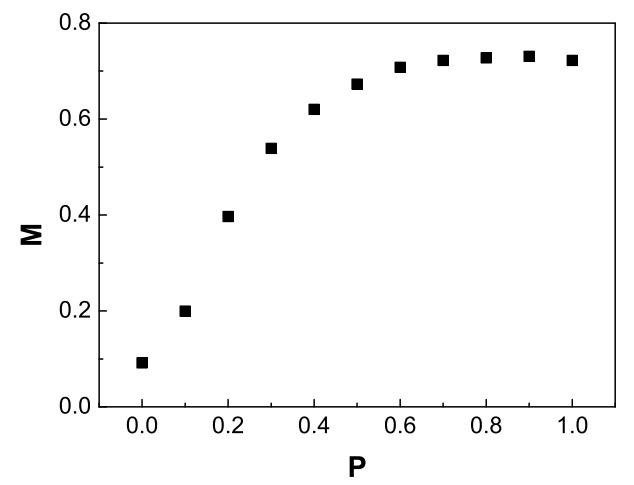

FIG. 4: Magnetization $M$ versus the probability $P$ of disordering link weights when $K_{B} T=14$.

fixed temperature is not obvious. We also make simulations on a larger ring of typically $N=1000$ vertices with $k=200$ neighbors per vertex. Figure 5 gives the curves of Magnetization $M$ as functions of the temperature and the disordering probability at fixed temperature. The systems of different sizes are ordered at different temperature $T$ as the ground state energy per spin diverges with the size $N$ increasing(as shown in Fig2(a) and Fig 5 (a)). This is not a key issue in our model as we only care about the influence of disordering link weights on the critical temperature of the same size systems. Fortunately, the systems with same parameters are exactly ordered at the same temperature. Obviously, the system is in the paramagnetic phase $(\langle M\rangle \approx 0)$ at $P=0$, and it is in the ordered, ferromagnetic phase $(|\langle M\rangle|>0.5)$ at $P=1$ (as shown in Fig $5(\mathrm{~b})$ ).

In all the simulations, the connecting structures of networks remain unchanged. The only factor, which can affect the behavior of phase transition, is the redistribution of link weights. This demonstrates that link weight plays an important role in the function of a network system.

We restrict the system size to $N$ in our studies, because a large number of configurations are required to get accurate results. The final results are the averages over 200 simulations, including 10 initial configurations of spins on the same network and 20 different networks generated with the same parameters. As the networks are densely connected, it consumes a lot of CPU time to search and update shortest paths. We have to limit our studies on relative small system size.

\section{CONCLUDING REMARKS}

As a measure of interaction strength, link weight is believed to be an important factor in networks. It gives more properties of the network besides topological structure, and provides an additional dimension to adjust network properties. For weighted networks, besides chang-
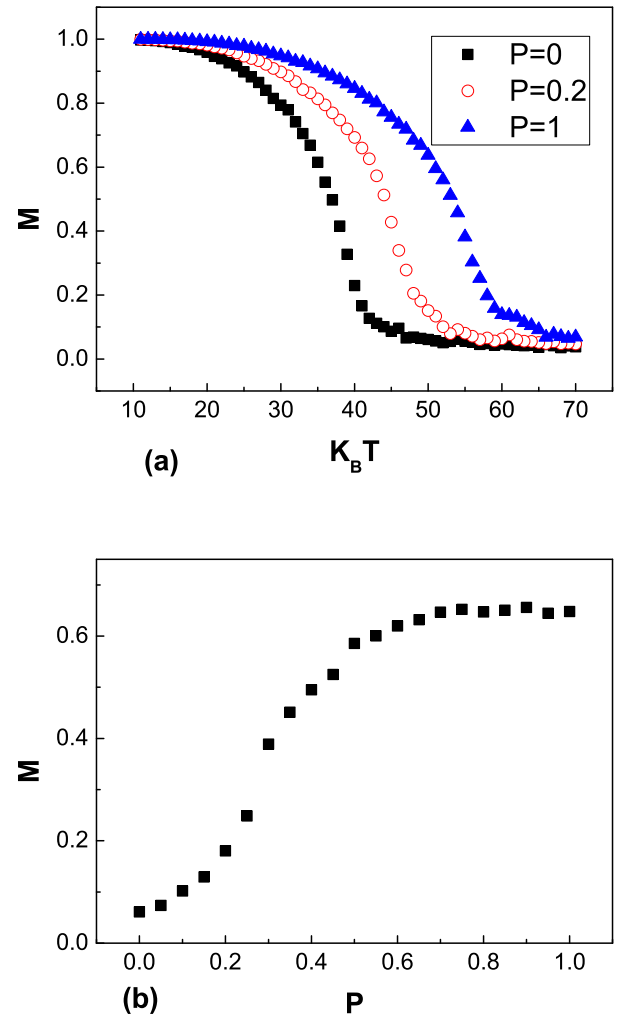

FIG. 5: Results in larger regular network with $N=1000$ and $k=200$. (a) Magnetization per spin $M$. (b)Magnetization versus the probability $P$ of disordering link weights when $K_{B} T=50$.

ing topology, redistributing link weights is an important way to optimize the dynamical behaviors of networks.

In this paper, we investigate the phase transition of the Ising model on weighted regular networks with interaction $J(d) \sim d^{-1}$. We have performed Monte Carlo simulations on networks with different probability $P$ of disordering link weights. The results show that disordering link weights can affect the critical temperature of phase transition. At fixed temperature, the order parameter $M$ attains the value $M \simeq 0$ for the paramagnetic phase at $P=0$ and a value $M>0$ for the ordered, ferromagnetic phase at $P=1$.

Although the effect of disordering weights is not so significant as that of changing topology, it is an important supplement way, and provide an additional approach to adjust the dynamical behaviors of the system.

This paper mainly focuses on the Ising model on weighted regular networks, where $J_{i j}=d_{i j}^{-\alpha}$ and $\alpha=1$. Of course, other value of $\alpha$ may be more useful to depict the effect of disordering link weights. Other dynamical process can also be investigated on weighted networks by similar approach. For further studies, the more interesting problem is to seek the best matching pattern between link weight and topological structure to optimize the dy- 
namical behaviors of the network. This would be very useful for designing networks and optimizing the dynamical behavior of the system.

\section{Acknowledgments}

Author M.H. Li wants to thank Xiaofeng Gong(NUS) for his reading and helpful comments. This work is par- tially supported by 985 Projet, NSFC under the grant No. 70771011 and No. 60974084.
[1] D. J. Watts and S. H. Strogatz, Nature 393440 (1998).

[2] L.F. Lago-Fernández, R. Huerta, F.Corbacho, and J. A. Sigüenza, Phys. Rev. Lett. 84 12(2000).

[3] R. Cohen, K. Erez, D. ben-Avraham, and S. Havlin, Phys. Rev. Lett. 854626 (2000).

[4] A. E. Motter and Y.-C. Lai, Phys. Rev. E 66 065102(R) (2002).

[5] A. Barrat and M. Weigt, Eur. Phys. J. B 13547 (2000).

[6] A. Pȩalski, Phys. Rev. E 64057104 (2001).

[7] S. Jespersen and A. Blumen, Phys. Rev. E 626270 (2000).

[8] R. Mousson, Eur. Phys. J. B 12, 555 (1999).

[9] P. Sen, K. Banerjee and T. Biswas, Phys. Rev. E. 66 037102 (2002).

[10] M. Gitterman, J. Phys. A 338373 (2000).

[11] C. P. Herrero, Phys. Rev. E 65066110 (2002).

[12] D. Jeong, H. Hong, B. J. Kim, and M. Y. Choi, Phys. Rev. E 68027101 (2003).

[13] P. K. Das and P. Sen, Eur. Phys. J. B 47 391-396 (2005).

[14] A. Chatterjee, P. Sen, Phys. Rev. E 74036109 (2006).

[15] S. Roy and S. M. Bhattacharjee, Phys. Lett. A 35213 (2006).

[16] C. V. Giuraniuc, J. P. L. Hatchett, J. O. Indekeu, M. Leone, I. Pérez Castillo, B. Van Schaeybroeck, and C. Vanderzande, Phys. Rev. E. 74036108 (2006).

[17] W. Li and X. Cai, Phys. Rev. E 69046106 (2004).

[18] A. Barrat, M. Barthélemy, R. Pastor-Satorras, A. Vespignani, Proc. Natl. Acad. Sci. U.S.A. 101(11) 3747-3752 (2004).

[19] M. E. J. Newman, Proc. Natl. Acad. Sci. U.S.A. 98404 (2001).

[20] M. E. J. Newman, Phys. Rev. E 64016131 (2001).

[21] Y. Fan, M. Li, J. Chen, L. Gao, Z. Di, J. Wu, International Journal of Modern Physics B Vol. 18, Nos. 17-19 2505-2511 (2004).
[22] E. Almaas, B. Kovacs,T. Vicsek and A.-L. Barabási, Nature, 427839 (2004).

[23] G. Yan, T. Zhou, J. Wang, Z.-Q. Fu, B.-H. Wang, Chin. Phys. Lett., Vol. 22, No. 2510 (2005).

[24] K. I. Goh, B. Kahng, D. Kim, Phys. Rev. E 72017103 (2005).

[25] K. I. Goh, J.D. Noh, B. Kahng, D. Kim, Phys. Rev. E 72017102 (2005).

[26] I. Vragović, E. Louis, and A. Díz-Guilera, Phys. Rev. E 71036122 (2005).

[27] G. Li, L. A. Braunstein, S. V. Buldyrev, S. Havlin, and H. E. Stanley, Phys. Rev. E. 75045103 (2007).

[28] M. Chavez, D.-U. Hwang, A. Amann, H. G. E. Hentschel, and S. Boccaletti, Phys. Rev. Lett. 94218701 (2005).

[29] C. Zhou and J. Kurths, Phys. Rev. Lett. 96164102 (2006).

[30] C. Zhou, A. E. Motter, and J. Kurths, Phys. Rev. Lett. 96034101 (2006).

[31] C. Zhou, L. Zemanová, G. Zamora, C. C. Hilgetag, and J. Kurths, Phys. Rev. Lett. 97238103 (2006).

[32] M. Li, Y. Fan, J. Chen, L. Gao, Z. Di, J. Wu, Physica A 350 643-656 (2005).

[33] M. Li, Y. Fan, D. Wang, D. Li, J. Wu, Z. Di, Physics Letters A 364 488-493 (2007).

[34] D. Li, M. Li, J. Wu, Z. Di, Y. Fan, Eur. Phys. J. B. 57, 423-428 (2007).

[35] J.-P. Onnela, J. Saramaki, J. Kertesz and K. Kaski, Phys. Rev. E 71065103 (2005).

[36] P. Holme, S. M. Park, B.J. Kim, C.R. Edling, Physica A 373 821-830 (2007).

[37] M.E. Fisher, S.-k. Ma, and B.G. Nickel, Phys. Rev. Lett. 29, 917 (1972).

[38] E. Luijten and H.W.J. Blote, Phys. Rev. Lett. 89, 025703 (2002). 